\title{
Data Collection and Application of COVID-19 in Medium-Sized Cities-Taking Macau as an Example
}

\author{
Yiyang Li, Ziyi Chen \\ Faculty of Innovation and Design, City University of Macau, Macau, China \\ Email: yiyangli327@gmail.com,czy20200316@163.com
}

How to cite this paper: Li, Y. Y., \& Chen, Z. Y. (2021). Data Collection and Application of COVID-19 in Medium-Sized Cities-Taking Macau as an Example. Current Urban Studies, 9, 574-580. https://doi.org/10.4236/cus.2021.93034

Received: August 23, 2021

Accepted: September 11, 2021

Published: September 14, 2021

Copyright ( 2021 by author(s) and Scientific Research Publishing Inc. This work is licensed under the Creative Commons Attribution International License (CC BY 4.0).

http://creativecommons.org/licenses/by/4.0/

\begin{abstract}
Convenient transportation and the large flow of residents in the city give rise to the wide spread of COVID-19. Data collection plays a crucial role in determining the effectiveness of the control of the pandemic. The pandemic is not only a serious problem in large cities, but also hitting medium-sized cities. Macau is a typical medium-sized city where the pandemic has been under effective control with comprehensive data collection. This thesis will conduct in-depth research on the collection and application of the statistics about COVID-19 in Macau. Macau collects data through the border, incorporating NAT (nucleic acid testing) data and health codes into daily-used mobile applications, which features a network with real-time updates and sharing. This thesis will connect to the construction of smart cities to provide ideas for handling large-scale public health issues in medium-sized cities.
\end{abstract}

\section{Keywords}

COVID-19, Data Collection, Smart City

\section{Introduction}

Existing epidemic prevention and control in the urban field mostly focuses on the construction of resilient cities, the impact on urban planning and design, and the research on emergency management countermeasures for urban public emergencies. Epidemic information at the city level is also an important aspect of epidemic prevention and control. Epidemic data is usually directly presented to the public. However, only a few studies have shown that on how urban individuals collect epidemic data. This article can fill the gap in this regard. This article uses Macau as a base. Macau has a special geographical location and environment. 
During the epidemic, there was a huge flow of people entering and exiting customs. This is very rare during the global anti-epidemic period. The article will analyze the characteristics of the spread of the epidemic in medium-sized cities, focusing on discovering how Macau collects and applies epidemic data, and in which aspects it has merits.

\section{Methods}

Use "COVID-19" or "Coronavirus" and "data collection" as keywords to conduct a quick review of the literature database of Web of Science, Scopus and CNKI. The latest information regarding data collection and application for COVID-19 is collected, and then analyzed to identify its potential application in pandemic prevention and control. Using COVID-19 data collection and application as keywords to search in Web of Science, 121 related articles can be obtained. Use data, collection, application, and epidemic as the title, abstract, and keywords of the paper to search in Scopus, and get 259 document search results. When you conduct an advanced search on CNKI with the topic of COVID-19, data, collection, and application, you can search 18 related articles.

\section{Risk of Spreading the Epidemic in Medium-Sized Cities}

The pandemic is raging around the world, not only in large cities, but also in medium-sized cities. According to China's Green Paper on Small and Mediumsized Cities, a city with the urban merits resident population of 500,000 to 1 million is regarded as medium-sized. The total permanent population of Macau in the fourth quarter of 2020 is 683.1 thousand, which is comparable to the population of many county-level cities in mainland China. According to statistics, China has 427 county-level cities, 337 prefecture-level cities, 4 municipalities and 3 special zones. The number of county-level cities accounts for $55 \%$ of the total number of Chinese cities. It can be viewed that the number of county-level cities accounts for half of the number of cities in China, and the spread of the virus will not stop due to differences in urban population and city sizes. Industry 4.0 is known as the fourth industrial revolution, which started with the innovation of advanced manufacturing and digital information technology. It is possible for such technology to meet customized requirements during the COVID-19 pandemic. However, on the front-line to first carry out the new industrial revolution are large-scale cities with more financial significance. The development of digital information technology in small and medium-sized cities is relatively slow (Javaid et al., 2020).

Medium-sized cities are smaller in size with a smaller population. They also face problems such as underdeveloped infrastructure and slow information transmission. The risk of the epidemic may not be smaller or even larger than that of large cities with a faster speed of transmission. Medium-sized cities often experience less access to information regarding the pandemic with the lack of effective communication on updates about virus transmission, making it more diffi- 
cult to control the pandemic (Figure 1).

\section{Data Collection and Application of Macau's Covid-19 Pandemic}

Macau had its first COVID-19 confirmed case at the end of January 2020. As of August2021, the total number of positive cases has reached 63 (Data source: Anti-epidemic page of Macao Health Bureau). The first ten cases of COVID-19 in Macau were imported cases, with seven from Wuhan for tourism purposes, who were diagnosed from the $22^{\text {nd }}$ to $27^{\text {th }}$ January 2020 . Their recorded dates of entry to Macau ranged from January $19^{\text {th }}$ to $23^{\text {rd }}, 2020$ (Lo et al., 2020).

Macau takes the lead in reducing cases to zero in the Guangdong-Hong KongMacao Greater Bay Area, and data collection and application have played a significant role in shaping preventive measures. Due to the special circumstances in Macau, a large number of people entry and exit the port each day, which brings a great risk of infection. Data collection is particularly important, and effective collection of data will offer help in contact tracing and clustering, screening and surveillance, and in reducing workload of medical staff (Swayamsiddha \& Mohanty, 2020).

The collection of Macau's Covid-19 pandemic data is a process of continuous optimization. On February 26, 2020, an electronic personal health declaration system was established. On May 3, 2020, the Macau Health Code was launched, which is an upgraded version of the personal health declaration. The code merges and replaces the existing health declaration system at the port of entry, leading to the closed loop management of entry to the community. The telephone hotline has been opened so that citizens can learn about the epidemic situation in

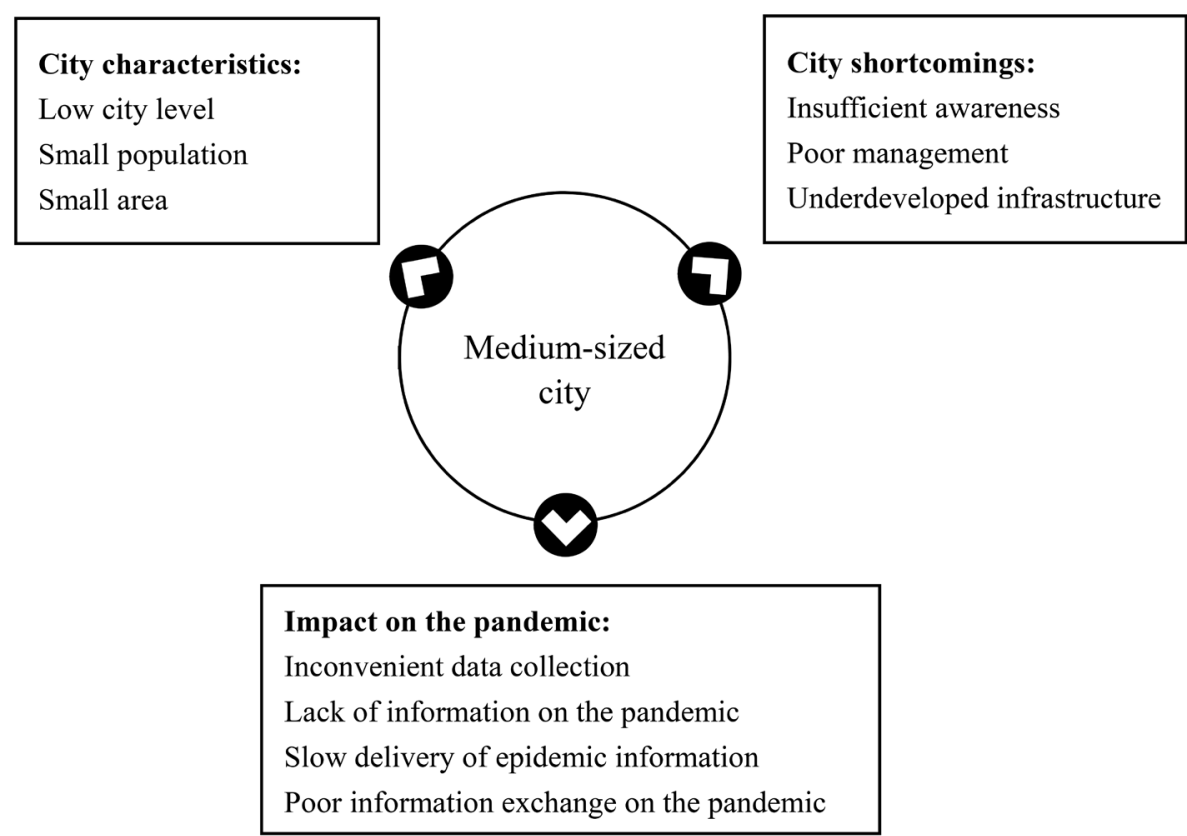

Figure 1. Characteristics of medium-sized cities. 
real time and report the epidemic information they have learned.

Macau collaborates with Guangdong government to integrate the Macau health code into WeChat for unified management. What's more, the code is connected to medical systems in Guangdong and Macau, which is presented in the customs clearance certificate. After the NAT (nucleic acid testing) is completed, the result will be directly displayed on the electronic customs clearance certificate on mobile devices. This ensures that all entry and exit personnel have tested negative for NAT. A large quantity of information has been collected through the network of border ports between Macau and Guangdong, which can be useful in pandemic prevention and control.

Macau has established a coordination center to update the Covid-19 pandemic situation and response measures in the form of text, illustrations, photos, videos and animations on websites, TV, radio, mainstream social platforms at home and abroad, etc., so that citizens can receive the information regarding the pandemic as soon as possible (Zheng \& Chen, 2020). Macau University of Science and Technology cooperates with the Hong Kong Polytechnic University and some domestic institutions to integrate epidemic information and develop an artificial intelligence (AI) system for rapid diagnosis of new cases. The application of this AI will contribute to early detection and prediction of COVID-19 based on regional information (Vaishya et al., 2020).

The specialized pandemic prevention page set up on the website of the Macao Health Bureau provides detailed data and information, including the latest news of the pandemic, announcements, pandemic prevention guidelines, testing statistics, pandemic prevention facilities, and vaccine information. Surveillance statistics are updated at $4 \mathrm{pm}$ every day, and the source, gender, age, date of diagnosis and patient status of the cases are recorded in detail. The website also marks all locations with stock of masks in Macau, including the address of the store, telephone number, and business hours. The data is updated every $15 \mathrm{mi}-$ nutes, so that the public can understand the supply of masks and choose the most convenient time and place to purchase (Figure 2).

\section{The Reference Effect of Macau Pandemic Information Collection for Medium-Sized Cities}

During the epidemic, big cities have advantage in data collection and application. Data is often integrated at the national level, but pandemic or large-scale infectious disease control is a decision at the local level, which requires more detailed data at the city level. In Mainland China, the data reflecting the pandemic situation is collected based on prefecture-level cities, while data on medium-sized cities is not reported in detail. On the one hand, medium-sized cities are not paid enough attention that the pandemic situation in big cities tends to receive more attention. On the other hand, due to inadequate management and underdeveloped communication infrastructure in medium-sized cities, it is also difficult to collect data in a timely and effective manner. The application of 


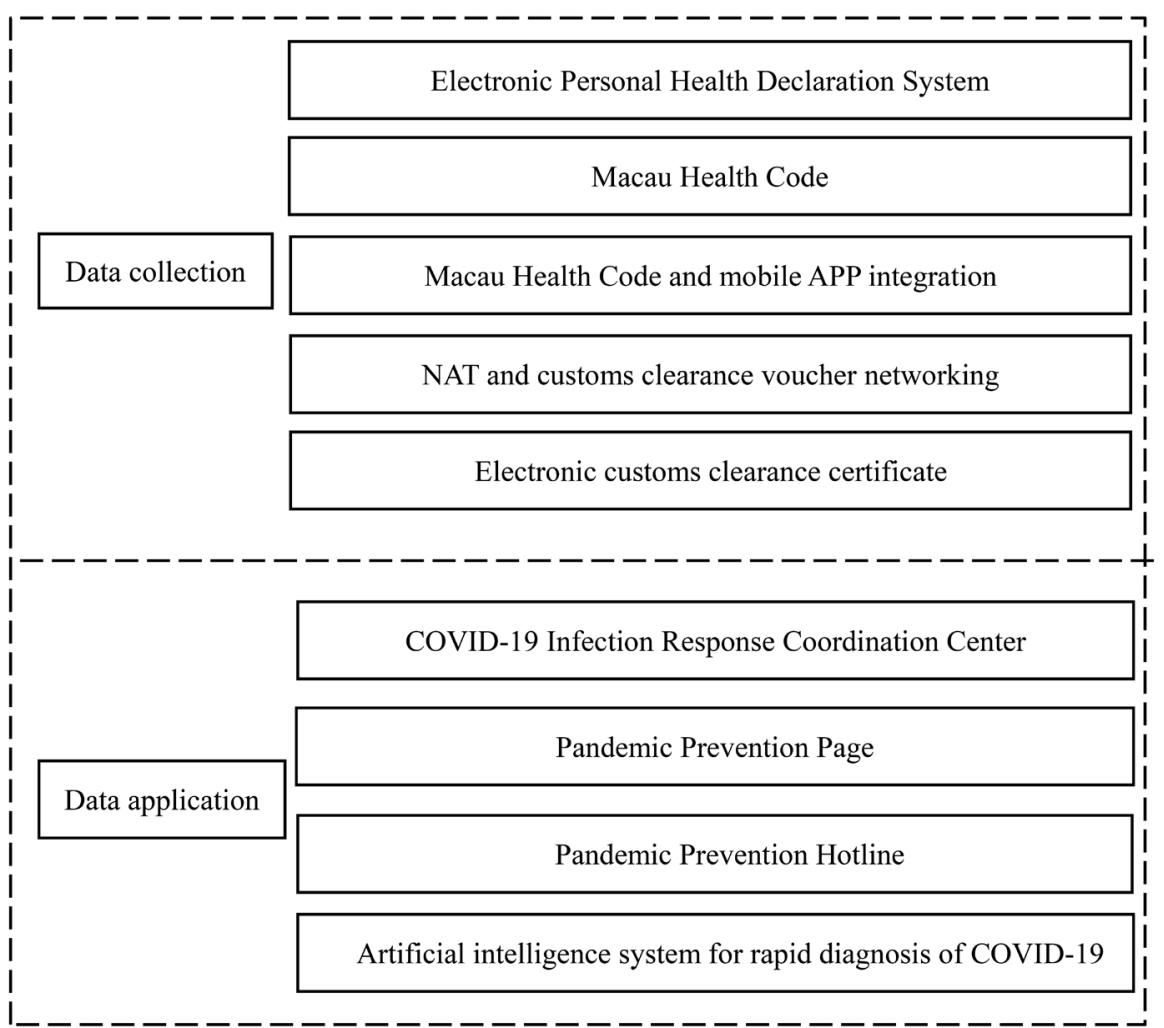

Figure 2. Data collection and application of Macau.

epidemic data in medium-sized cities often relies on large cities, and health codes are shared with large cities. The epidemic data of large cities can be specific to the district, while the epidemic data of medium-sized cities are included under the data of large cities, and it is difficult to form its own epidemic prevention and control system. When an epidemic occurs, the source of the data must also be collected and applied through a higher-level system, which will make it difficult for medium-sized cities to respond to the epidemic in a timely manner, making it difficult to adapt to local conditions and effectively control their own actual conditions. In summary, it is difficult for medium-sized cities to collect information during the pandemic and use it effectively, which adversely affects the control of the pandemic.

The pandemic in Macau has been well controlled, which can be used as a reference for medium-sized cities with a population range from 500,000 to 1 million. First, the pandemic data needs to be collected from the city borders. The entrance and exit ports of Macau are ideal places for data collection. Mediumsized cities have the characteristics of smaller size and area with limited transport access to the city, which makes it convenient to carry out data collection at the borders of the city. Passengers' health information can be embedded into the vehicle's ETC (Electronic Toll Collection) card, and body temperature can be directly tested when vehicles are passing through the ETC scan. Second, medium-sized cities can set up separate websites like Macau to update data in real 
time and display all data on websites, TV, radio, and mobile applications, and present the result of NAT along with health condition on mobile software. Mobile technology on its own and as extension of telemedicine serves a significantly constructive role in the current COVID-19 pandemic (Iyengar et al., 2020).

The medium-sized city is small in size and communities are closely connected. At the same time, marking the location where cases are spotted can effectively reduce the possibility of the citizen's infection at the same place, as well as help them make judgment of their health conditions.

\section{Conclusion}

The COVID-19 pandemic poses new challenges to the development of cities. In the construction of smart cities, more emphasis is placed on the collection and application of data, which should apply to cities regardless of sizes. As a case of a medium-sized city in China, Macau has a high population density and high mobility, but the epidemic has been well controlled, which is inseparable from its detailed data collection and data application in place. In the future, the construction of cities is not only about the rise of buildings. The establishment of largescale digital "new infrastructure" (Wu et al., 2021), detailed collection of various data and application of various types of urban problems in accordance with local conditions are the top priority of smart city construction.

In 2021, the epidemic is not over. The new type of COVID-19 is still circulating worldwide and has mutated into the Delta virus. The collection of epidemic data is gradually normalizing. Mutated viruses spread faster and face greater challenges for the epidemic in medium-sized cities. The data of medium-sized cities and large cities in epidemic prevention and control are scattered and fragmented. It is difficult to apply them according to their own conditions, and the epidemic prevention measures still have to refer to the regulations formulated by higherlevel cities. In the post-epidemic era, data is more diversified, and data is more scattered and random. Medium-sized cities not only rely on the support of large cities to form their own systems for data collection and application. As a relatively independent individual city, Macau, on the one hand, cooperates with the large-scale system of Guangdong, but also has its own data sources and data application systems, has good resistance to the epidemic.

\section{Conflicts of Interest}

The authors declare no conflicts of interest regarding the publication of this paper.

\section{References}

Iyengar, K., Upadhyaya, G. K. et al. (2020). COVID-19 and Applications of Smartphone Technology in the Current Pandemic. Diabetes \& Metabolic Syndrome: Clinical Research \& Reviews, 14, 733-737. https://doi.org/10.1016/j.dsx.2020.05.033

Javaid, M., Haleem, A. et al. (2020). Industry 4.0 Technologies and Their Applications in 
Fighting COVID-19 Pandemic. Diabetes \& Metabolic Syndrome: Clinical Research \& Reviews 14, 419-422. https://doi.org/10.1016/j.dsx.2020.04.032

Lo, I. L. et al. (2020). Evaluation of SARS-CoV-2 RNA Shedding in Clinical Specimens and Clinical Characteristics of 10 Patients with COVID-19 in Macau. International Journal of Biological Sciences, 16, 1698-1707. https://doi.org/10.7150/ijbs.45357

Swayamsiddha, S., \& Mohanty, C. (2020). Application of Cognitive Internet of Medical Things for COVID-19 Pandemic. Diabetes \& Metabolic Syndrome: Clinical Research \& Reviews, 14, 911-915. https://doi.org/10.1016/j.dsx.2020.06.014

Vaishya, R., Javaid, M. et al. (2020). Artificial Intelligence (AI) Applications for COVID-19 Pandemic. Diabetes \& Metabolic Syndrome: Clinical Research \& Reviews, 14, 337-339. https://doi.org/10.1016/j.dsx.2020.04.012

$\mathrm{Wu}, \mathrm{Z}$. Q. et al. (2021). On the Iterative Law of New Infrastructure Construction. Urban Planning, 45, 9-14.

Zheng, L., \& Chen, Y. L. (2020). Analyze Macau City During Public Health Emergencies and Countermeasures. International Journal of Social Science and Education Research 3, 168-177. 